\title{
INFLUENCE OF FIELD CROP ROTATIONS ON THE BIOLOGICAL ACTIVITY OF PEAT SOILS
}

\author{
V. A. Pronevych
}

Institute of Agroecology and Environmental Management, NAAS, Kyiv

12, Metrolohichna Str., Kyiv City, 03143, Ukraine e-mail: vasyl.pronevych@mail.ru

To preserve the fertility and productivity of drained peat soils it is important to develop scientifically grounded crop rotation, selection and rational cropping sequence, regulation of the processes of organic matter mineralization taking into account the requirements of environmental protection. In connection with the aggravation of environmental situation soils that are influenced by intensive human activities deserve special attention, in which, due to insufficient scientific substantiation of processes, profound changes of ground take place, the deterioration of their ecological condition is observed [1-3].

Biological activity of the soil is a combination of biological processes that occur in the soil as a result of life activity of soil microorganisms. The ecological and phytosanitary state of soils is largely dependent on the structure of microbial communities, interaction of their basic components. Decomposition of plant and animal residues, release and binding of nutrition elements is also a specialized function of microorganisms [4].

There are close links between the microbiota in the soil. Change of activity of population of one species affects the activity of the other. Free-existing nitrogen fixers are provided with nitrogen but require a source of carbon.

Cellulose-decomposing microorganisms, on the contrary, receive carbon from decomposed organic matter, but are dependent on external supply of nitrogen. Research has confirmed close interaction of the representatives of cellulose-decomposing and nitrogen fixing microorganisms in field conditions. In the soil with stubble, which is a substrate for the development of cellulosolithic bacteria and micromycetes, higher nitrogen activity is observed than in the soil without plant residues [5].

For optimal functioning of soil, and any other, ecosystem maintaining close relationships of microorganisms is necessary. Soil microorganisms undergo seasonal dynamics; depend on soil type, climate zone and so on. Therefore, each agrocenosis is characterized by individual biological activity. Due to microbiological activity changes in the structure of soil and micromorphological structure occur, resulting in the improvement of plants nutrient status and moisture provision during the growing season [7; 8].

Numerous studies have proved that plants affect life activity of other organisms of the soil. The roots of plants alter soil structure, air treatment, are involved in the decomposition of minerals, and are the source of organic matter. Biotic relationships constantly occur between plants and microorganisms which are reflected on the increase in the number of members of the separate physiological groups of microorganisms in rhizospheric area. Root secretions of plants specifically affect both the development of root zone microorganisms and their activity. Plants biomass is also the source of organic matter in the soil [9-11].

The objective of our research was to study the orientation and intensity of microbiological processes in drained peat soils at their use in crop rotations compared with long-term meadows.

Materials and methods. The study was conducted during 1986-2006 on marshy area "Chemerne" of Sarny scientific and research station for the development of marshland of the Institute of Water Problems and Land Reclamation of NAAS. 11 schemes of the most effective in the conditions of Woodlands nine-field arable and fodder crop rotations, the structure of cropping areas, cultivation and fertilization systems, aimed to preserve peat soils were studied in the stationary experiment. Recommended amounts of fertilizers for drained peat soils were applied under crops at the rate of

$\mathrm{R}_{30-60} \mathrm{~K}_{90-150}$. The results were compared with the area of natural long-term meadows where intensive anthropogenic interference was not conducted. The level of groundwater in the 
experiment ranged under perennial grass in April at the rate of $40 \mathrm{~cm}$, in September - 105 $\mathrm{cm}$, under cultivated grass -30 and $80 \mathrm{~cm}$, under grain crops -30 and $90 \mathrm{~cm}$ respectively. In some years groundwater level decreased to $120 \mathrm{~cm}$.

Technologies and research methods generally accepted in soil science, forage manufacture and agrochemical practice were used in the experiment. Biological activity of soil was determined by the intensity of cloth applications decomposition within 60-day period in the layer of $0-30 \mathrm{~cm}$; nitrate nitrogen - by Grandval-Lajoux with the use of phenoldisulfonic acid; ammonia nitrogen in $1 \mathrm{~N}$ potassium chloride with Nessler reagent.

Statistical data processing was performed by analysis-of-variance method by
Dospiekhov (1985) using MS Excel 2003 and «Statistics».

So, the ecological state of drained peat soils is determined by the level of soil biota activity, which in turn depends on the way of management. Introduction of grain-grass crop rotations on peat soils with the share of perennial grasses at the rate of $66-77 \%$ contributes to relatively high microbiological activity of ploughed layer, organic matter preservation, fully meets crops requirements of mineral forms of nitrogen that mostly approximates the functioning of agroecosystems to their natural counterparts. Intensive use of peat soils in tilled crop rotation leads to excessive microbiological activity and rapid decomposition of organic matter of peat deposits. 